\title{
A Numerical Study of Surface Waves of Finite Amplitude.
}

\author{
By
}

\section{Koji Hidaka and Akira NaKano}

\section{Introduction.}

The determination of the wave forms satisfying the conditions of uniform propagation without change of type was worked out by Stokes (1847) and others. Stokes obtained the equation of the free surface of waves in terms of a stream function (Lamb 1932), and expanded this into an infinite series using successive approximations. Thus he could determine the wave form when the steepness does not exceed a certain limit. The purpose of the present research is to find out wave forms from Stokes' equation by means of numerical calculations instead of Stokes' expansion with successive approximations.

\section{Calculations.}

With Stokes let us assume that the water is incompressible, and that the motion is restricted in two dimensions. Thus there exists a stream function $\psi(x, y)$ which satisfies Laplace's differential equation. We further add the backward velocity $-C$, which is equal and in opposite direction to the phase velocity of the original waves, by which the motion of water can be made stationary. In this case, the profile of the free surface must evidently be a streamline; we take it to be the line: $\psi=0$. Hence, in order to determine the wave forms, we have only to find $\psi$ which satisfies Laplace's equation and the condition of uniform pressure along the free surface. Such a function $\psi$ was found by Stokes, or

$$
\frac{\psi}{C}=-y+\beta e^{k y} \cos k x
$$

retaining the first term of expansion only. For the equation of the free surface $\psi=0$, we have, then

$$
y=\beta e^{k y} \cos k x
$$

which gives the wave profile on the surface. Stokes expanded the above equation into an infinite series by successive approximations, and obtained the following result :

$$
y=\text { const. }+a \cos k x-\left(\frac{1}{2} k a^{2}+\frac{17}{24} k^{3} a^{4}\right) \cos 2 k x+\ldots \ldots
$$

By this series he could calculate the wave profile for the waves whose steepness does not exceed a certain limit. Now in this paper an atempt is to be made to find the wave forms from (2) by means of a numerical method. 
From (2) we have

$$
-k y+k \beta e^{k y} \cos k x=0
$$

which becomes

$$
-\zeta+X e^{\zeta} \cos \xi=0
$$

on putting

$$
\xi=k x, \quad \zeta=k y \quad \text { and } \quad X=k \beta .
$$

For any assigned value of $X$, equation (5) gives a curve, which can be determined by trial and errors. But if we change $X$ to another value which does not differ much from the first, the approximate value of $\zeta$ for any value of $\xi$ can be fonnd very quickly. In this research we carried out the calculations

\begin{tabular}{|c|c|c|c|c|c|c|c|}
\hline$X=$ & 0.06256 & 0.07801 & 0.10374 & 0.15305 & 0.20039 & 0.28744 & $1 / e$ \\
\hline$\xi$ & $\zeta$ & $\zeta$ & $\zeta$ & $\zeta$ & $\zeta$ & $\zeta$ & $\zeta$ \\
\hline 0 & 0.06689 & 0.08499 & 0.11622 & 0.18396 & 0.25985 & 0.45145 & 1.00000 \\
\hline $\pm 1 \pi / 18$ & 0.06579 & 0.08352 & 0.11423 & 0.17963 & 0.25456 & 0.43885 & 0.83509 \\
\hline $\pm 2 \pi / 18$ & 0.06259 & 0.07940 & 0.10836 & 0.17057 & 0.23918 & 0.40562 & 0.68853 \\
\hline $\pm 3 \pi / 18$ & 0.05738 & 0.07265 & 0.09892 & 0.15472 & 0.21431 & 0.35503 & 0.55492 \\
\hline $\pm 4 \pi / 18$ & 0.05040 & 0.06369 & 0.08641 . & 0.13406 & 0.18464 & 0.29606 & 0.43570 \\
\hline $\pm 5 \pi / 18$ & 0.04193 & 0.05286 & $0.07 \mathrm{~J} 44$ & 0.10980 & 0.14960 & 0.23331 & 0.32839 \\
\hline $\pm 6 \pi / 18$ & 0.03231 & 0.04063 & 0.05465 & 0.08317 & 0.11208 & 0.17042 & 0.23196 \\
\hline $\pm 7 \pi / 18$ & 0.02187 & 0.02763 & 0.03671 & 0.05532 & 0.07379 & 0.10971 & 0.14553 \\
\hline $\pm 8 \pi / 18$ & 0.01098 & 0.01374 & 0.01821 & 0.02732 & 0.03608 & 0.05261 & 0.06841 \\
\hline $\pm 9 \pi / 18$ & 0.00000 & 0.00000 & 0.00000 & 0.00000 & 0.00000 & 0.00000 & 0.00000 \\
\hline $\pm 10 \pi / 18$ & -0.01074 & -0.01337 & -0.01766 & -0.02590 & -0.03365 & -0.04759 & -0.06015 \\
\hline $\pm 11 \pi / 18$ & -0.02096 & -0.02619 & -0.03420 & -0.04980 & -0.06438 & -0.08986 & -0.11244 \\
\hline $\pm 12 \pi / 18$ & -0.03035 & -0.03757 & -0.04925 & -0.07127 & -0.09144 & -0.12633 & -0.15719 \\
\hline $\pm 13 \pi_{j} 18$ & -0.03869 & -0.04783 & -0.06248 & -0.08992 & -0.11484 & -0.15779 & -0.19460 \\
\hline $\pm 14 \pi / 18$ & -0.04578 & -0.05648 & -0.07363 & -0.10550 & -0.13423 & -0.18331 & -0.22503 \\
\hline $\pm 15 \pi / 18$ & -0.05152 & -0.06341 & -0.08251 & -0.11781 & -0.14945 & -0.20316 & -0.24849 \\
\hline $\pm 16 \pi / 18$ & -0.05561 & -0.06846 & -0.08895 & -0.12671 & -0.16040 & -0.21734 & -0.26517 \\
\hline $\pm 17 \pi / 18$ & -0.05813 & -0.07153 & -0.09286 & -0.13208 & -0.16700 & -0.22585 & -0.27515 \\
\hline $\pm 18 \pi / 18$ & -0.05898 & -0.07255 & -0.09417 & -0.13387 & -0.16920 & -0.22868 & -0.27847 \\
\hline$\delta$ & 0.02003 & 0.02507 & 0.03348 & 0.05058 & 0.06829 & 0.10824 & 0.20347 \\
\hline
\end{tabular}
for various values of $X$, and determined the wave forms as are seen in Table $I$.

Table I.

By $\delta=\left(\zeta_{\text {max }}-\zeta_{\text {min }}\right) / \lambda$, it will be possible for us to know the ratio of wave height to wave length, usually called the steepness. It is seen from this analysis that the value of $\delta$ increases as $X$ is increased until at last the profile of the wave will have a cusp at its crest. 


\section{Remarks on the Steepest Wave.}

It is shown by hydrodynamics that the steepest wave has a crest angle $120^{\circ}$ and a steepness $1 / 7$. Let us try to compute this limiting case. Let the coordinates of the top of the crest and the corresponding limiting value of $X$ be $\xi_{0}, \zeta_{0}$ and $X_{0}$ respectively. Since the velocity must vanish at the top, we have the following conditions:

$$
\begin{aligned}
& \left(\frac{\partial \psi}{\partial y}\right)_{\xi_{0}, \zeta_{0}}=0 \quad \therefore-1+X_{0} e^{\xi_{0}} \cos \xi_{0}=0, \\
& \left(\frac{\partial \psi}{\partial x}\right)_{\xi_{0}, \zeta_{0}}=0 \quad \therefore-X_{0} e_{0} \sin \xi_{0}=0 .
\end{aligned}
$$

Thus we have

$$
\xi_{0}=0, \quad X_{0}=\frac{1}{e^{\zeta_{0}}} .
$$

As this point lies on the surface, $\xi_{0}, \zeta_{0}$ and $X_{0}$, of course, satisfy (5). Thus we have

$$
-\zeta_{0}+X_{0} e^{\zeta_{0}} \cos \xi_{0}=0
$$

From (6) and (7) we have

$$
\xi_{0}=0, \quad \zeta_{0}=1, \quad X_{0}=\frac{1}{e} .
$$

From (4) and (8) we have the equation of the limiting wave form, viz.

$$
-\zeta+e^{(\zeta-1)} \cos \xi=0 \text {. }
$$

If $X$ is larger than $1 / e$, we can easily prove that (5) cannot have any real root for $\xi=0$.

Now we have from (9)

$$
\frac{d \zeta}{d \xi}=\frac{e^{(\zeta-1)} \sin \xi}{1-e^{(\zeta-1)} \cos \xi}= \pm \frac{\sqrt{e^{2(\zeta-1)}-\zeta^{2}}}{1-\zeta},
$$

Hence, we have

$$
\begin{aligned}
\lim _{\zeta \rightarrow 1} \frac{d \zeta}{d \xi} & = \pm \lim _{\varepsilon \rightarrow 1} \frac{\sqrt{e^{-\varepsilon}-(1-\varepsilon)^{2}}}{\varepsilon}= \pm \lim _{\varepsilon \rightarrow 0} \frac{\sqrt{\left\{1-2 \varepsilon+1 / 2 !(2 \varepsilon)^{2}-\cdots\right\}-\left(1-2 \varepsilon-\varepsilon^{2}\right)}}{\varepsilon} \\
& = \pm 1 .
\end{aligned}
$$

This means that the angle of the crest in the limiting case is $90^{\circ}$ instead of $120^{\circ}$ obtained by Stokes. Numerical computation gives the steepness of the wave in this case $0.203 \ldots . .$. instead of the Michell's value 1/7 (J. H. Michell 1893). This discrepancy, of course, results from the fact that we only retained the first term in the solution of Laplace's equation as is seen in (1). 


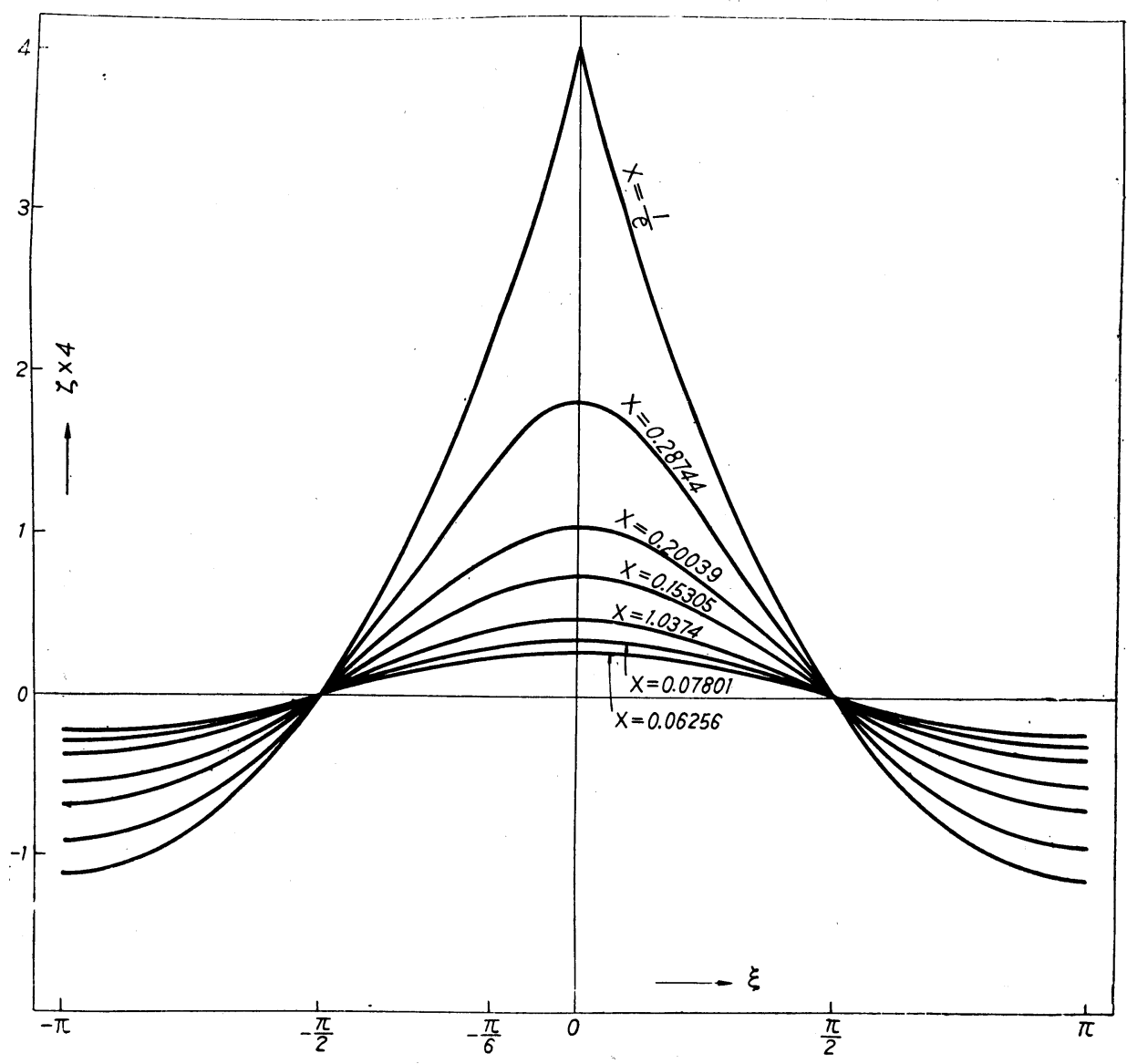

\section{References}

Lamb, Horace (1932): Hydrodynamics.

Michell, J.H. (1893) : "The highest waves in water," Phil. Mag. (5) xxxvi, 430.

Stokes, G. G. (1847) : “On the theory of oscillatory waves," Camb. Phil. Soc. Trans. viii, 441. 